\title{
Article \\ Molecular Determination of mirRNA-126 rs4636297, Phosphoinositide-3-Kinase Regulatory Subunit 1-Gene Variability rs7713645, rs706713 (Tyr73Tyr), rs3730089 (Met326Ile) and Their Association with Susceptibility to T2D
}

\author{
Rashid Mir ${ }^{1, *(D)}$, Imadeldin Elfaki ${ }^{2, *}$, Faisel M. Abu Duhier ${ }^{1, *}$, Maeidh A. Alotaibi ${ }^{3}$ (D) Adel Ibrahim AlAlawy ${ }^{2}$, \\ Jameel Barnawi ${ }^{1}$, Abdullatif Taha Babakr ${ }^{4}$, Mohammad Muzaffar Mir ${ }^{5}$ (D) Hyder Mirghani ${ }^{6}$, Abdullah Hamadi ${ }^{1}$ \\ and Pradeep Kumar Dabla ${ }^{7}$
}

1 Department of Medical Lab Technology, Faculty of Applied Medical Sciences, University of Tabuk, Tabuk 71491, Saudi Arabia; jbarnawi@ut.edu.sa (J.B.); a.aldhafri@ut.edu.sa (A.H.)

2 Department of Biochemistry, Faculty of Science, University of Tabuk, Tabuk 71491, Saudi Arabia; aalalawy@ut.edu.sa

3 King Faisal Medical Complex Department of Training, Research and Academic Affairs, P.O. Box 2775, Taif 21944, Saudi Arabia; maed96@hotmail.com

check for

updates

Citation: Mir, R.; Elfaki, I.; Duhier, F.M.A.; Alotaibi, M.A.; AlAlawy, A.I.; Barnawi, J.; Babakr, A.T.; Mir, M.M.; Mirghani, H.; Hamadi, A.; et al.

Molecular Determination of mirRNA-126 rs4636297,

Phosphoinositide-3-Kinase

Regulatory Subunit 1-Gene

Variability rs7713645, rs706713

(Tyr73Tyr), rs3730089 (Met326Ile) and Their Association with Susceptibility to T2D. J. Pers. Med. 2021, 11, 861. https://doi.org/10.3390/jpm11090861

Academic Editor:

Konstantinos Tziomalos

Received: 11 August 2021

Accepted: 27 August 2021

Published: 29 August 2021

Publisher's Note: MDPI stays neutral with regard to jurisdictional claims in published maps and institutional affiliations.

Copyright: (c) 2021 by the authors. Licensee MDPI, Basel, Switzerland. This article is an open access article distributed under the terms and conditions of the Creative Commons Attribution (CC BY) license (https:// creativecommons.org/licenses/by/ $4.0 /)$.
4 Department of Medical Biochemistry, Faculty of Medicine, Umm Al-Qura University, Makkah 57039, Saudi Arabia; abdullatiftaha@yahoo.com

5 Department of Basic Medical Sciences, College of Medicine, University of Bisha, Bisha 61992, Saudi Arabia; mirmuzaffar11@gmail.com

6 Internal Medicine and Endocrine, Medical Department, Faculty of Medicine, University of Tabuk, Tabuk 71491, Saudi Arabia; h.mirghani@ut.edu.sa

7 Department of Biochemistry, Govind Ballabh Pant Institute of Postgraduate Medical Education \& Research (GIPMER), Associated to Maulana Azad Medical College, Delhi 110002, India; pradeep.dabla@gmail.com

* Correspondence: rashidmirtabuk@gmail.com (R.M.); ielfaki@ut.edu.sa (I.E.); fabu-duhier@ut.edu.sa (F.M.A.D.)

Abstract: Type 2 diabetes is a metabolic disease characterized by elevated blood sugar. It has serious complications and socioeconomic impact. The MicroRNAs are short single-stranded and non-coding RNA molecules. They regulate gene expression at the post-transcriptional levels. They are important for many physiological processes including metabolism, growth, and others. The phosphoinositide 3-kinase (PI3K) is important for insulin signaling and glucose uptake. The genome wide association studies have identified the association of certain loci with diseases including T2D. In this study we have examined the association of miR126 rs4636297 and Phosphoinositide-3-kinase regulatory subunit 1 (PIK3R1) gene Variations rs7713645, rs706713 (Tyr73Tyr), and rs3730089 (Met326Ile) with T2D using the amplification refractory mutation system PCR. Results indicated that there was a significant different ( $p$-value < 0.05) in the Mir126 rs4636297 genotypes distribution between cases and controls, and the minor allele of the rs4636297 was also associated with T2D with OR $=0.58$, $p$-value $<0.05$. In addition results showed that there were significant differences $(p$-value $<0.05)$ of rs4636297 genotype distribution of patients with normal and patient with abnormal lipid profile. Results also showed that the PIK3R1 rs7713645 and rs3730089 genotype distribution was significantly different between cases and controls with a $p$-values $<0.05$. In addition, the minor allele of the rs7713645 and rs3730089 were associated with T2D with OR $=0.58, p$-value $<0.05$. We conclude that the Mir126 rs4636297 and PIK3R1 SNPs (rs7713645 and rs3730089) were associated with T2D. These results need verification in future studies with larger sample sizes and in different populations. Protein-protein interaction and enzyme assay studies are also required to uncover the effect of the SNPs on the PI3K regulatory subunit (PI3KR1) and PI3K catalytic activity.

Keywords: diabetes mellitus; genome wide association studies; microRNAs; gene variations; miR126 rs4636297; phosphoinositide 3-kinase (PI3K) 


\section{Introduction}

Diabetes mellitus (DM) is a group of metabolic disorders characterized by increased blood glucose due to insufficient insulin amount or defective insulin action or both [1]. DM is one of the global health issues due to its bad socio-economic effects on the patients, their families and public health [2]. The incidence rate of DM has risen in most countries. It has been estimated in year 2017 that there were more than 400 million individuals suffering from DM [2]. This number is expected to rise to more than 690 million by the year 2045 if no sufficient preventive and control measures were adopted [2]. In term of DM prevalence, the WHO has ranked KSA as number two in the Middle East with seven million individuals suffering from DM and 3 million were reported to be pre-diabetics [3]. The long-term complications of DM include microvascular and macro vascular complications [4]. The microvascular complications include the diabetic nephropathy (DN), diabetic retinopathy (DR) and diabetic neuropathy (DN) [4], whereas the macrovascular complications are the cardiovascular diseases such as coronary artery disease, stroke and peripheral artery disease [4]. Mainly, there are two main types of DM, type 1 DM (T1DM) and type 2 DM (T2DM). T1DM is developed from destruction of pancreatic beta cells by cellular mediated autoimmune process leading to absolute insulin deficiency and represent from 5 to $10 \%$ of DM [1,5]. T2DM is induced from defective insulin signaling initiated by insulin resistance in liver, adipose tissues and skeletal muscles. This leads to defective insulin metabolic action and relative insulin deficiency [6]. T2DM is developed by the interaction of environmental and genetic risk factors [7]. Environmental and risk factors of T2DM include aging, obesity, physical inactivity, unhealthy diet [7]. Genome wide association studies (GWAS) have revealed the association of certain loci with the risk to several diseases including DM [8-14]. MicroRNAs (miRNAs) are short (22 nucleotides length) single-stranded and non-coding RNA molecules. They regulate gene expression at the post-transcriptional levels [15]. MiRNAs regulate physiological process e.g., metabolism, development, proliferation, differentiation and apoptosis [15]. It has been reported that in T2D there was a reduced plasma level of mir126, and there would be loss of endothelial miR-126 [16]. Furthermore, reduced plasma miR-126 has been suggested as a biomarker for T2DM [17]. In addition, the miR-126 rs4636297 SNP was associated with DR in T1DM patients from Brazil [18]. PI3K is a heterodimeric enzyme composed of a catalytic subunit (p110) and a regulatory subunit (p85) [19]. The PI3K regulatory subunit (PIK3R1) comprised a SH3 domain, a breakpoint cluster region homology domain, two SH2 domains, and an inter-SH2 domain [20]. The PI3K is an important in insulin signaling cascade [21], and the PI3K/AKT signaling pathway is important for normal metabolism and its dysregulation results in obesity and T2DM [22]. In the present study we evaluated the association of miR126 (rs4636297) and PIK3R1 gene variations rs7713645, rs706713 (Tyr73Tyr), and rs3730089 (Met326Ile) with T2D.

\section{Materials and Methods}

This study was approved by the research and studies department, directorate of health affairs, Taif, approval No. 229, and by the research ethics committee of the armed forces hospitals, northwestern region, Tabuk, approval No. R \& REC2016-115. The study was conducted in the Department of Biochemistry, Faculty of Science in collaboration with Prince Fahd Bin Sultan Research Chair, Department of Medical Lab Technology, Faculty of Applied Medical Sciences, University of Tabuk. All subjects completed the questionnaire as well as informed consent.

\subsection{Data Collection}

This is a case-control study enrolled about 100 subjects each with type 2 diabetes mellitus (T2D) and about 120 normal control subjects for each SNP. T2D was diagnosed on the basis of the WHO criteria. This study included clinically confirmed cases of T2D in Saudi Arabia visiting the armed forces hospital in Tabuk, Al Noor Specialist Hospital in Mecca and the King Faisal Hospitals in Taif for routine checkup. The control subjects were 
matched healthy volunteers with no history of diabetes or any major clinical disorders and had normal fasting plasma glucose level. The T1D, T2D cases with other significant chronic diseases or malignancies were excluded from the study. The variables that were analyzed from the T2D patients include the case history, age and gender; duration of T2D; glycated hemoglobin (HBA1c); random blood glucose, total cholesterol, Triacylglycerol, high-density lipoprotein-Cholesterol (HDL-C), and low-density lipoprotein cholesterol (LDL-C) concentrations and total cholesterol/HDL-C ratios have been assayed using the standard protocols. The biochemical characteristics of control and cases were shown in Table 1.

Table 1. The glycated hemoglobin (HBA1c), Triglyceride (TG), cholesterol (choles.), low density lipoprotein cholesterol (LDL-C), high density lipoprotein cholesterol (HDL-C), fasting blood sugar (FBS), random blood sugar (RBS) and vitamin D of healthy controls and T2D patients.

\begin{tabular}{cccccccc}
\hline & HBA1c $\%$ & TG mg/dL & Choles. mg/dL & LDL-C mg/dL & HDL-C mg/dL & FBS mg/dL \\
\hline Controls & 5 & 135 & 153 & 74 & 57.0 & 89 & \\
\hline & HBA1c $\%$ & TG mg/dL & Choles mg/dL & LDL-C mg/dL & HDL-C mg/dL & RBS mg/dL & Vit. D ng/mL \\
\hline Cases & 9 & 178 & 198 & 130 & 44 & 224 & 28 \\
\hline
\end{tabular}

\subsection{Sample Collection and DNA Extraction}

From each Subject about $4 \mathrm{~mL}$, a peripheral blood sample was collected in an EDTA tube. Genomic DNA was isolated using the Thermo Scientific Genomic DNA Purification Kit (Waltham, MA, USA) from the whole blood according to the manufacturer's instructions. The DNA integrity was checked with 0.8 agarose gel electrophoresis and Nanodrop.

\subsection{Genotyping of SNPs by Amplification-Refractory Mutation System PCR}

The microR-126 rs4636297 A > G SNP was genotyped by ARMS-PCR (Figure 1A). The genotyping of three SNP of PIK3R1 gene, the PIK3R1 rs7713645 $A \rightarrow C$ (Figure 1B), PIK3R1 rs706713 C $\rightarrow$ T (Figure 1C) and PIK3R1 rs3730089 A $\rightarrow$ G (Figure 1D) by ARMS-PCR. The primers for all four SNPs were designed by using primer3 software as depicted in Table 2. The ARMS-PCR was done in a reaction volume of $25 \mu \mathrm{L}$ containing template DNA (50 ng), FO $-0.30 \mu \mathrm{L}, \mathrm{RO}-0.30 \mu \mathrm{L}, \mathrm{RI}-0.20 \mu \mathrm{L}, \mathrm{RI}-0.20 \mu \mathrm{L}$ of 25 pmol of each primers and $10 \mu \mathrm{L}$ from GoTaq ${ }^{\circledR}$ Green Master Mix (cat no M7122) (Promega, Madison, WI, USA). The final volume of $25 \mu \mathrm{L}$ was adjusted by adding nuclease free $\mathrm{dd}_{2} \mathrm{O}$. Finally, the $2 \mu \mathrm{L}$ of DNA was added from each patient. The thermocycling conditions used were at $95^{\circ} \mathrm{C}$ for $10 \mathrm{~min}$ followed by 40 cycles of $95^{\circ} \mathrm{C}$ for $35 \mathrm{~s}$, annealing temperature PIK3R1 rs706713 $\mathrm{C} \rightarrow \mathrm{T}\left(58^{\circ} \mathrm{C}\right)$ PIK3R1 rs3730089 $\mathrm{A} \rightarrow \mathrm{G}\left(60^{\circ} \mathrm{C}\right)$, PIK3R1 rs7713645 $\mathrm{A} \rightarrow \mathrm{C}\left(55^{\circ} \mathrm{C}\right)$ and microR-126 rs $4636297 \mathrm{~A}>\mathrm{G}\left(58^{\circ} \mathrm{C}\right)$ for $40 \mathrm{~s}, 72{ }^{\circ} \mathrm{C}$ for $43 \mathrm{~s}$ followed by the final extension at $72{ }^{\circ} \mathrm{C}$ for $10 \mathrm{~min}$. PCR products were separated on $2 \%$ agarose gel stained with $2 \mu \mathrm{L}$ of sybre safe stain and visualized on a UV transilluminator from Biorad (Hercules, CA, USA). 


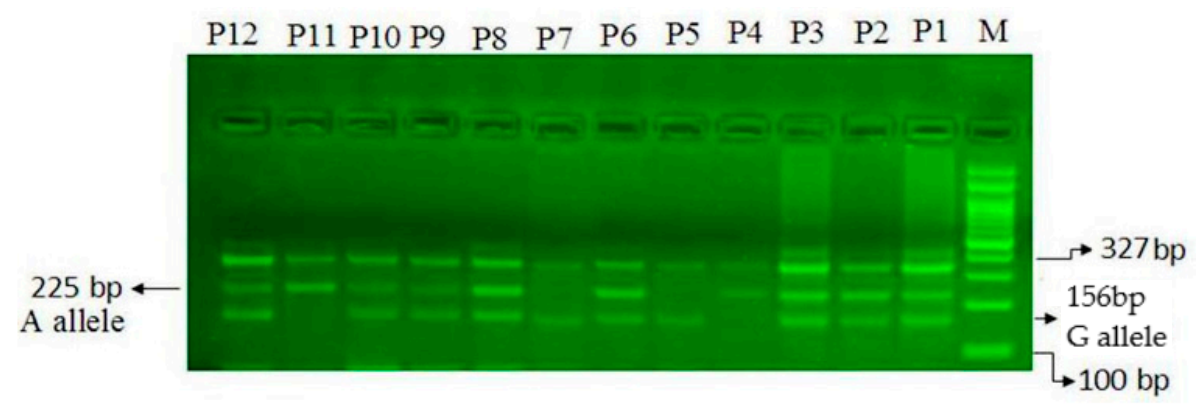

M: 100 bp ladder.

Heterozygous AG: P1, P2, P3, P6, P8, P9, P10, P12.

Homozygous AA: P4, P11

Homozygous GG: P5, P7

(A)

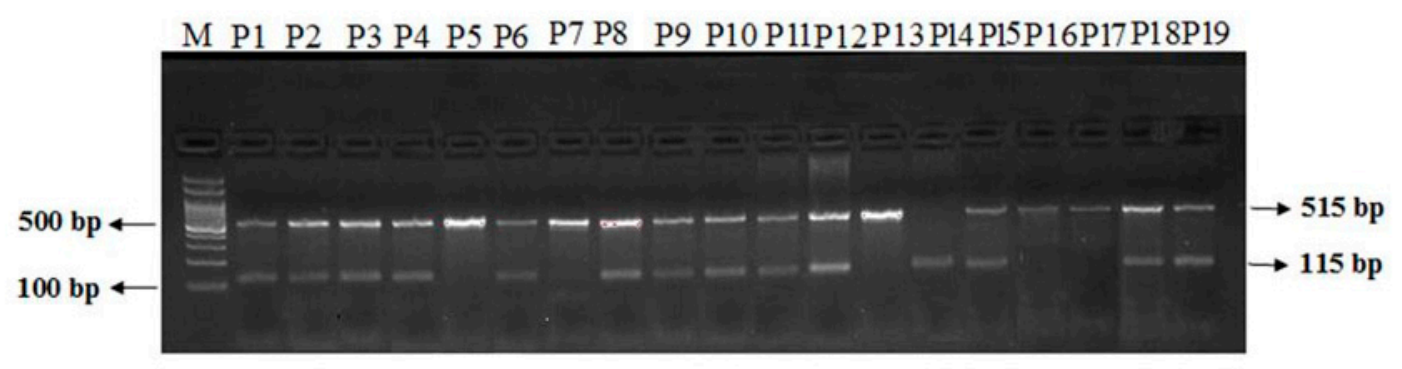

Legend

M: 100 bp DNA ladder.

Heterozygous genotype CA: P1, P2, P3, P4, P6, P8, P9, P10, P11, P12, P14, P15, P18 \& P19 T2D

Homozygous genotype AA: P14

Homozygous genotype CC: P5, P7, P13, P16 and P17.

(B)

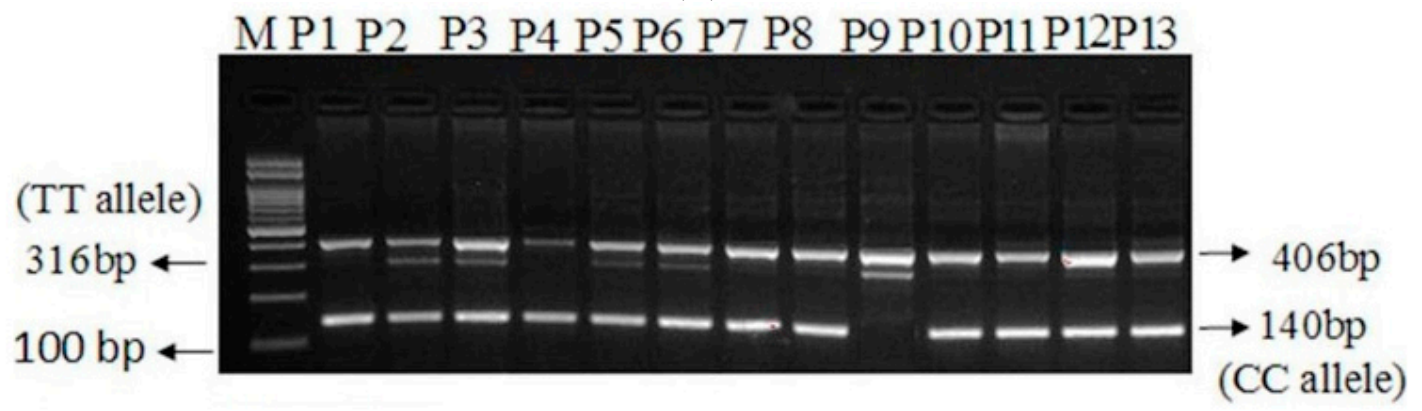

Legend

M-100 bp DNA ladder

Heterozygous: CT: P2, P3, P5, P6.

Homozygous: TT-P9

Homozygous: CC-P1, P4, P7, P8, P10, P11, 12, P13

(C)

Figure 1. Cont. 


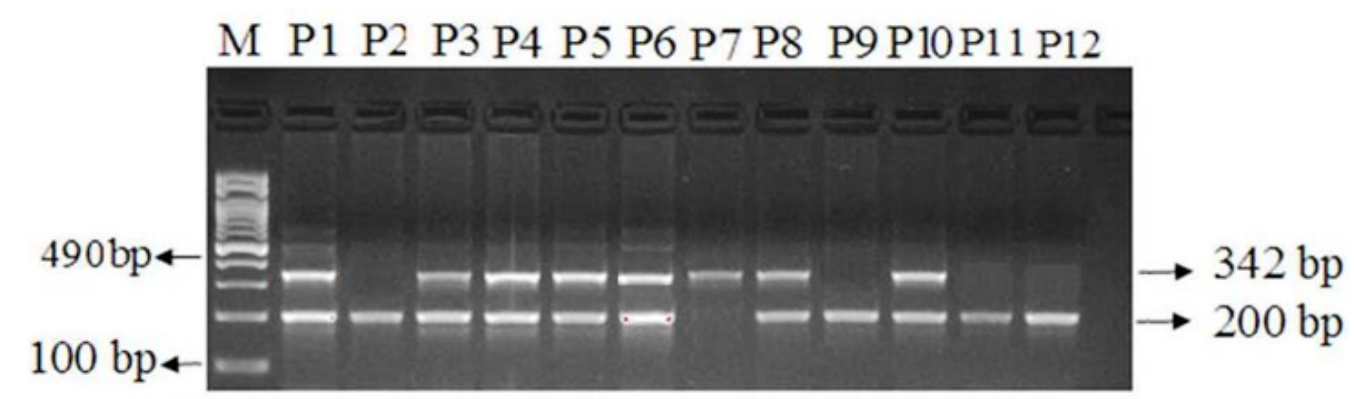

\author{
Legend \\ M-100 bp DNA ladder \\ Heterozygous GA: P1, P3, P4, P5, P6, P8, P10 \\ Homozygous AA: P2, P9, P11, P12 \\ Homozygous GG: P7
}

(D)

Figure 1. (A) Genotyping of the Mir126 rs4636297 using the ARMS-PCR, (B) Genotyping of the PIK3R1 rs7713645 using the ARMS-PCR, (C) Genotyping of the PIK3R1 rs706713 (Tyr73Tyr) using the ARMS-PCR, (D) Genotyping of the PIK3R1 rs3730089 (Met326Ile) using the ARMS-PCR.

Table 2. Primer sequence of microR-126 SNP and PIK3R1 gene SNPs.

\begin{tabular}{|c|c|c|c|}
\hline \multicolumn{4}{|c|}{ Primer Sequence of microR-126 rs4636297 A > G SNP } \\
\hline mi126Fo & 5-GGATAGGTGGGTTCCCGAGAACTG-3 & $327 \mathrm{bp}$ & $58^{\circ} \mathrm{C}$ \\
\hline mi126Ro & 5-TCTCAGGGCTATGCCGCCTAAGT-3 & & \\
\hline mi126FI-G & 5-TTCAAACTCGTACCGTGAGTAATAATGAGC-3 & $156 \mathrm{bp}$ & \\
\hline mi126RI-A & 5-GTTTTCGATGCGGTGCCGTGGAAGA-3 & $225 \mathrm{bp}$ & \\
\hline \multicolumn{4}{|c|}{ Primer Sequence of PIK3R rs7713645 A > C SNP } \\
\hline PIK3R1-F1 & 5-ССТАСАССААССССАТTCAGC-3 & $518 \mathrm{bp}$ & $58^{\circ} \mathrm{C}$ \\
\hline PIK3R1-A & 5-ACACTCAAATGCTGAATGTGAAAAGTT-3 & & \\
\hline PIK3R-F2 & 5-GGTTTCCCAAGGCATGTTATTGTCAC-3 & $115 \mathrm{bp}$ & \\
\hline PIK3R-R2C & 5-TAGTCAATGTTTGTGATTTATTGCAGCC-3 & & \\
\hline \multicolumn{4}{|c|}{ Primer Sequence of PIK3R rs706713 C > T SNP } \\
\hline PIK3R1-Fo & 5-TAAAAACGTAAAATCAGACTGCTCTG-3 & $406 \mathrm{bp}$ & $58^{\circ} \mathrm{C}$ \\
\hline PIK3R1-Ro & 5-TGACCTTGTTGTTCAACATCTGC-3 & & \\
\hline PIK3R1C-FI & 5-GGGACTTTCCGGGAACTTAC-3 & $140 \mathrm{bp}$ & \\
\hline PIK3R1T-RI & 5-GAGATTTTTTTCСТTCCAATATATTCTACA-3 & $316 \mathrm{bp}$ & \\
\hline \multicolumn{4}{|c|}{ Primer Sequence of PI3KR1 rs3730089 G > A SNP } \\
\hline PIK3R-F1 & CATGGCCAGCCCAATTTATTTGTTC & $490 \mathrm{bp}$ & $60^{\circ} \mathrm{C}$ \\
\hline PIK3R-R & CGTCTTTGGAAGAGAACCAACTATG & & \\
\hline PIK3R-F1A & GCCAACAACGGTATGAATAACAATA & $200 \mathrm{bp}$ & \\
\hline PIK3RI-C & GTACCATTCAGCATCTTGTAAGGAC & $342 \mathrm{bp}$ & \\
\hline
\end{tabular}

\title{
3. Statistical Analysis
}

T2D patients and controls were compared by statistical analysis using the SPSS 16.0 software package. Chi-square analysis and Fisher exact test were carried out to compare microR-126 rs4636297 A > G, PIK3R1 rs706713 C $\rightarrow$ T, PIK3R1 rs3730089 A $\rightarrow$ G, PIK3R1 rs7713645 $\mathrm{A} \rightarrow \mathrm{C}$ gene polymorphism frequency with several clinical aspects. The Hardy- 
Weinberg equilibrium was tested by a $\chi^{2}$ test to compare the observed genotype frequencies within the case-control groups. $p$-value was considered to be significant when it was $<0.05$.

\section{Results}

Results indicated that there was a significant difference in Mir126 rs4636297 genotypes (AA, AG, GG) distribution between cases $(65,43,05)$ and controls $(47,54,13)$ with $p$-value $=0.021$ (Table 3). In the co-dominant model, it was shown that the GG genotype was associated with T2D with an $\mathrm{OR}(95 \% \mathrm{CI})=\mathrm{O} .27(0.09$ to 0.83$)$, $\mathrm{RR}=0.58(0.40$ to 0.83$)$, $p$-value $=0.02$ (Table 4). In the dominant model, $(\mathrm{GA}+\mathrm{GG})$ was associated with T2D with an OR $(95 \% \mathrm{CI})=0.51(0.30-0.87), \mathrm{RR}=0.72(0.55-0.94), p$-value $=0.014$ (Table 4$)$. The $\mathrm{G}$ allele was also associated with T2D with $\mathrm{OR}(95 \% \mathrm{CI})=0.58(0.38-0.87), \mathrm{RR}=0.77$ $(0.64-0.93), p$-value $=0.009$ (Table 4). In addition, results showed that there were significant differences ( $p$-values $<0.05$ ) in the lipid profile in patients with normal and patient with abnormal lipid profile (Table 5).

Table 3. Association of MicroRNA-126 rs4636297 A > G genotypes between T2D patients and healthy controls.

\begin{tabular}{cccccccccc}
\hline Subjects & $\boldsymbol{n}$ & AA & GA & GG & A & G & $\chi^{2}$ & df & $p$-Value \\
\hline $\begin{array}{c}\text { T2D } \\
\text { patients }\end{array}$ & 113 & $65(57.52 \%)$ & $43(38 \%)$ & $05(4.42 \%)$ & 0.77 & 0.23 & 7.69 & 2 & $\mathbf{0 . 0 2 1}$ \\
\hline Controls & 114 & $47(41.22 \%)$ & $54(47.36 \%)$ & $13(11.40 \%)$ & 0.65 & 0.35 & & & \\
\hline
\end{tabular}

Statistically significant $p$-values $(p<0.05)$ were indicated with bold numbers.

Table 4. Association of MicroRNA-126 rs4636297 A > G gene variation with T2D.

\begin{tabular}{|c|c|c|c|c|c|}
\hline $\begin{array}{c}\text { Mode of } \\
\text { Inheritance }\end{array}$ & $\begin{array}{l}\text { Controls } \\
(n=114)\end{array}$ & $\begin{array}{c}\text { Cases } \\
(n=113)\end{array}$ & OR $(95 \% \mathrm{CI})$ & RR (95\% CI) & $p$-Value \\
\hline \multicolumn{6}{|l|}{$\begin{array}{l}\text { Co-dominant } \\
\text { model }\end{array}$} \\
\hline MiR-AA & 47 (41.22\%) & $65(57.52 \%)$ & 1 (ref.) & 1 (ref.) & \\
\hline MiR-GA & $54(47.36 \%)$ & $43(38 \%)$ & 0.57 (0.33 to 0.99$)$ & $0.76(0.57$ to 1.00$)$ & 0.05 \\
\hline MiR-GG & $13(11.40 \%)$ & $05(4.42 \%)$ & 0.27 (0.09 to 0.83$)$ & $0.58(0.40$ to 0.83$)$ & 0.02 \\
\hline \multicolumn{6}{|l|}{ Dominant model } \\
\hline MiR-AA & 47 (41.22\%) & $65(57.52 \%)$ & 1 (ref.) & 1 (ref.) & \\
\hline $\operatorname{MiR}(\mathrm{GA}+\mathrm{GG})$ & 67 (58.77\%) & 48 (42.47\%) & $0.51(0.30-0.87)$ & $0.72(0.55-0.94)$ & 0.014 \\
\hline \multicolumn{6}{|l|}{ Recessive model } \\
\hline $\operatorname{MiR}(\mathrm{AA}+\mathrm{GA})$ & $101(88.59 \%)$ & $108(95.57 \%)$ & 1 (ref.) & 1 (ref.) & \\
\hline MiR-GG & $13(11.40 \%)$ & $05(4.42 \%)$ & $0.35(0.12-1.04)$ & $0.69(0.48-0.92)$ & 0.067 \\
\hline \multicolumn{6}{|l|}{ Allele } \\
\hline MiR-A & 148 & 173 & 1 (ref.) & 1 (ref.) & \\
\hline MiR-G & 78 & 53 & $0.58(0.38-0.87)$ & $0.77(0.64-0.93)$ & 0.009 \\
\hline
\end{tabular}

Abbreviations: OR = Odds Ratio, RR = Risk Ratio, $\mathrm{CI}=$ Confidence interval; Statistically significant $p$-values $(p<0.05)$ were indicated with bold numbers. 
Table 5. Clinical associations of MicroRNA rs4636297 G > A genotypes with clinic variables features of the T2D patients.

\begin{tabular}{|c|c|c|c|c|c|c|c|}
\hline Subjects & $n=113$ & AA & GA & GG & $x^{2}$ & df & $p$-Value \\
\hline \multicolumn{8}{|l|}{ Association with gender } \\
\hline Males & 80 & 50 & 26 & 04 & 3.6 & 2 & 0.160 \\
\hline Females & 33 & 15 & 17 & 01 & & & \\
\hline \multicolumn{8}{|l|}{ Association with Age } \\
\hline Age $>20$ & 27 & 15 & 10 & 02 & 0.75 & 2 & 0.068 \\
\hline Age $>40$ & 86 & 50 & 33 & 03 & & & \\
\hline \multicolumn{8}{|c|}{ Association with RBS mg/dL } \\
\hline RBS $<140$ & 34 & 22 & 10 & 02 & 1.62 & 2 & 0.444 \\
\hline RBS > 140 & 79 & 43 & 33 & 03 & & & \\
\hline \multicolumn{8}{|c|}{$\begin{array}{l}\text { Association with Cholesterol } \\
\mathrm{mg} / \mathrm{dL}\end{array}$} \\
\hline Cholesterol $<200$ & 81 & 50 & 30 & 01 & 7.54 & 2 & 0.023 \\
\hline Cholesterol > 200 & 32 & 15 & 13 & 04 & & & \\
\hline \multicolumn{8}{|c|}{ Association with HDL-C mg/dL } \\
\hline HDL-C $<55$ & 79 & 48 & 30 & 01 & 6.4 & 2 & 0.048 \\
\hline HDL-C > 55 & 34 & 17 & 13 & 04 & & & \\
\hline \multicolumn{8}{|c|}{ Association with LDL-C mg/dL } \\
\hline LDL $<100$ & 23 & 06 & 15 & 04 & 26.1 & 2 & 0.0001 \\
\hline LDL > 100 & 77 & 59 & 28 & 01 & & & \\
\hline \multicolumn{8}{|c|}{ Association with TG mg/dL } \\
\hline TG $<200$ & 61 & 47 & 13 & 01 & 20.87 & 2 & 0.0001 \\
\hline TG $>200$ & 52 & 18 & 30 & 04 & & & \\
\hline \multicolumn{8}{|c|}{ Association with HBA1c \% } \\
\hline HBA1c $<6$ & 27 & 15 & 10 & 2 & 0.25 & 2 & 0.882 \\
\hline HBA1c $>6$ & 86 & 50 & 33 & 3 & & & \\
\hline \multicolumn{8}{|c|}{ Association with Vitamin D ng/mL } \\
\hline Vit.D < 30 & 18 & 03 & 13 & 02 & 2.54 & 2 & 0.28 \\
\hline Vit.D > 30 & 14 & 1 & 13 & 0 & & & \\
\hline
\end{tabular}

Statistically significant $p$-values $(p<0.05)$ were indicated with bold numbers.

Results showed that there was a significant difference in PI3KR1 rs7713645 genotype (AA, AC, CC) distribution between cases $(08,80,12)$ and controls $(37,60,11)$ with a $p$-value $=0.0001$ (Table 6). Results also showed that in the Co-dominant model, the CA genotype is associated with T2D with an $\mathrm{OR}=6.16$ (2.67 to 14.20$)$, $\mathrm{RR}=1.91(1.51$ to 2.42 ), $p$-value $=0.0001$ (Table 7). In the dominant model, the (CA + CC) was associated with T2D with an OR $=5.99(2.62-13.66), \mathrm{RR}=1.88(1.51-2.35), p$-value $=0.0001$ (Table 6). Moreover, the $\mathrm{C}$ allele was associated with T2D with $\mathrm{OR}=1.77(1.19-2.61), \mathrm{RR}=1.32(1.08-1.60)$, $p$-value $=0.004$ (Table 7). Moreover, our result showed that there were significant differences ( $p$-value $<0.05)$ in the lipid profile in patients with normal and patient with abnormal lipid profile (Table 8). Results indicated there was a significant difference in the genotype distribution between patients with age over 20 and less than 40 years old. 
Table 6. Association of PIKR rs7713645 A > C genotypes between T2D patients and healthy controls.

\begin{tabular}{|c|c|c|c|c|c|c|c|c|c|}
\hline Subjects & $n$ & AA & CA & $\mathrm{CC}$ & A & $\mathrm{C}$ & $x^{2}$ & df & $p$-Value \\
\hline $\begin{array}{l}\text { PIKR } \\
\text { patients }\end{array}$ & 100 & $8(8 \%)$ & $80(80 \%)$ & $12(12 \%)$ & 0.48 & 0.52 & 21.31 & 2 & 0.0001 \\
\hline Controls & 108 & 37 (34.25\%) & $60(55.55 \%)$ & $11(10.18 \%)$ & 0.62 & 0.38 & & & \\
\hline
\end{tabular}

Table 7. Association of PI3KR1 rs7713645 A > C gene variation with T2D.

\begin{tabular}{|c|c|c|c|c|c|}
\hline $\begin{array}{c}\text { Mode of } \\
\text { Inheritance }\end{array}$ & Controls $(n=108)$ & $\begin{array}{c}\text { Cases } \\
(n=100)\end{array}$ & OR $(95 \%$ CI $)$ & RR $(95 \%$ CI) & $p$-Value \\
\hline \multicolumn{6}{|l|}{$\begin{array}{l}\text { Co-dominant } \\
\text { model }\end{array}$} \\
\hline PIKR-AA & 37 & 8 & 1 (ref.) & 1 (ref.) & \\
\hline PIKR-CA & 60 & 80 & $6.16(2.67$ to 14.20$)$ & 1.91 (1.51 to 2.42$)$ & 0.0001 \\
\hline PIKR-CC & 11 & 12 & 5.04 (1.64 to 15.45$)$ & 1.71 (1.09 to 2.69$)$ & 0.0046 \\
\hline \multicolumn{6}{|l|}{ Dominant model } \\
\hline PIKR-AA & 37 & 8 & 1 (ref.) & 1 (ref.) & \\
\hline PIKR-(CA + CC) & 71 & 92 & $5.99(2.62-13.66)$ & $1.88(1.51-2.35)$ & 0.0001 \\
\hline \multicolumn{6}{|l|}{ Recessive model } \\
\hline PIKR-(AA + CA) & 97 & 88 & 1 (ref.) & 1 (ref.) & \\
\hline PIKR-CC & 11 & 12 & $1.20(0.50-2.86)$ & $1.09(0.70-1.71)$ & 0.67 \\
\hline \multicolumn{6}{|l|}{ Allele } \\
\hline PIKR-A & 134 & 96 & 1 (ref.) & 1 (ref.) & \\
\hline PIKR-C & 82 & 104 & 1.77 (1.19-2.61) & $1.32(1.08-1.60)$ & 0.004 \\
\hline
\end{tabular}

Abbreviations: $\mathrm{OR}=$ Odds Ratio, $\mathrm{RR}=$ Risk Ratio, $\mathrm{CI}=$ Confidence interval; Statistically significant $p$-values $(p<0.05)$ were indicated with bold numbers.

Table 8. Clinical associations of PI3KR1 rs7713645 A > C SNP genotypes with clinic variables features of the T2D patients.

\begin{tabular}{|c|c|c|c|c|c|c|c|}
\hline Subjects & $n=100$ & AA & CA & $\mathrm{CC}$ & $x^{2}$ & df & $p$-Value \\
\hline Association with gender & & 8 & 80 & 12 & & & \\
\hline Males & 59 & 4 & 47 & 8 & 0.56 & 2 & 0.755 \\
\hline Females & 41 & 4 & 33 & 4 & & & \\
\hline Association with Age & & 8 & 80 & 12 & & & \\
\hline Age $>20$ & 14 & 04 & 08 & 02 & 9.75 & 2 & 0.0076 \\
\hline Age $>40$ & 86 & 04 & 72 & 10 & & & \\
\hline Association with RBS mg/dL & & 8 & 80 & 12 & & & \\
\hline RBS $<140$ & 34 & 05 & 27 & 02 & 4.5 & 2 & 0.102 \\
\hline RBS $>140$ & 66 & 03 & 53 & 10 & & & \\
\hline Association with Cholesterol mg/dL & & 8 & 80 & 12 & & & \\
\hline Cholesterol < 200 & 64 & 05 & 60 & 02 & 16.14 & 2 & 0.0003 \\
\hline Cholesterol $>200$ & 36 & 3 & 20 & 10 & & & \\
\hline
\end{tabular}


Table 8. Cont.

\begin{tabular}{|c|c|c|c|c|c|c|c|}
\hline Subjects & $n=100$ & AA & CA & $\mathrm{CC}$ & $x^{2}$ & df & $p$-Value \\
\hline Association with HDL-C mg/dL & & 8 & 80 & 12 & & & \\
\hline $\mathrm{HDL}<55 \mathrm{mg}$ & 72 & 05 & 63 & 04 & 11.7 & 2 & 0.0039 \\
\hline $\mathrm{HDL}>55 \mathrm{mg}$ & 28 & 03 & 17 & 08 & & & \\
\hline \multicolumn{8}{|l|}{ Association with LDL-C mg/dL } \\
\hline LDL-C $<100$ & 23 & 05 & 14 & 04 & 9.14 & 2 & 0.010 \\
\hline LDL-C > 100 & 77 & 03 & 66 & 08 & & & \\
\hline \multicolumn{8}{|l|}{ Association with TG mg/dL } \\
\hline TG $<200$ & 73 & 03 & 62 & 08 & 6.31 & 2 & 0.045 \\
\hline TG $>200$ & 27 & 05 & 18 & 04 & & & \\
\hline \multicolumn{8}{|l|}{ Association with HBA1c \% } \\
\hline HBA1c $<6$ & 01 & 0 & 1 & 0 & 0.25 & 2 & 0.882 \\
\hline HBA1c $>6$ & 99 & 08 & 79 & 12 & & & \\
\hline \multicolumn{8}{|l|}{ Association with Vitamin D ng/mL } \\
\hline Vit.D $<30$ & 18 & 03 & 13 & 02 & 2.54 & 2 & 0.28 \\
\hline Vit.D $>30$ & 14 & 1 & 13 & 0 & & & \\
\hline
\end{tabular}

Statistically significant $p$-values $(p<0.05)$ were indicated with bold numbers.

Results showed that there was a significant differences in the rs706713 SNP genotype (CC, CT, TT) distribution between the cases $(68,33,0)$, and controls $(53,45,03)$ with $p$-value $=0.03$ (Table 9). Results showed that in the dominant model, the (TC + TT) was associated with OR $(95 \% \mathrm{CI})=0.53(0.3-0.94), \mathrm{RR}=0.73(0.56-0.96), p$-value $=0.032$ (Table 10). Furthermore, the T allele was associated with T2D with OR $(95 \% \mathrm{CI})=0.36$ $(0.22-0.58), \mathrm{RR}=0.59(0.47-0.73), p$-value $=0.0001$ (Table 10). Results indicated there was a significant difference in the genotype distribution between patients with aged over 20 and patients aged less than 40 years old (Table 11).

Table 9. Genotype frequency of PI3KR1 SNP rs706713 T > C polymorphism of study cohorts.

\begin{tabular}{lcccccc}
\hline Variables/Genotype & C/C & T/C & T/T & $\chi^{2}$ & df & $p$-Value \\
\hline PIKR patients & $68(67.3 \%)$ & $33(32.7 \%)$ & $0(0 \%)$ & 6.71 & 2 & $\mathbf{0 . 0 3}$ \\
\hline Controls & $53(52.5 \%)$ & $45(44.6 \%)$ & $3(2.9 \%)$ & & & \\
\hline
\end{tabular}

Statistically significant $p$-values $(p<0.05)$ were indicated with bold numbers.

Table 10. Association of PI3KR1 SNP rs706713 C > T gene variation with PIKR.

\begin{tabular}{cccccc}
\hline $\begin{array}{c}\text { Mode of } \\
\text { Inheritance }\end{array}$ & $\begin{array}{c}\text { Cases } \\
(\boldsymbol{n = 1 0 1 )}\end{array}$ & $\begin{array}{c}\text { Controls } \\
(\boldsymbol{n}=\mathbf{1 0 1})\end{array}$ & OR (95\% CI) & RR (95\% CI) & $\boldsymbol{p}$-Value \\
\hline Co-dominant & 68 & 53 & 1 (ref.) & 1 (ref.) & 0.056 \\
\hline PIKR-CC & 33 & 45 & $0.57(0.32-1.01)$ & $0.75(0.57-1.0)$ & 0.149 \\
\hline PIKR-TC & 00 & 03 & $0.11(0.005-2.2)$ & $0.43(0.35-0.53)$ & \\
\hline PIKR-TT & & & & 1 (ref.) \\
\hline Dominant & 68 & 53 & & 1 (ref.) & \\
\hline PIKR-CC & & &
\end{tabular}


Table 10. Cont.

\begin{tabular}{|c|c|c|c|c|c|}
\hline $\begin{array}{c}\text { Mode of } \\
\text { Inheritance }\end{array}$ & $\begin{array}{c}\text { Cases } \\
(n=101)\end{array}$ & $\begin{array}{l}\text { Controls } \\
(n=101)\end{array}$ & OR $(95 \% \mathrm{CI})$ & RR (95\% CI) & $p$-Value \\
\hline PIKR-(TC + TT) & 33 & 48 & $0.53(0.3-0.94)$ & $0.73(0.56-0.96)$ & 0.032 \\
\hline \multicolumn{6}{|l|}{ Recessive } \\
\hline PIKR-(CC + TC) & 101 & 98 & 1 (ref.) & 1 (ref.) & \\
\hline PIKR-TT & 00 & 03 & $0.13(0.007-2.7)$ & $0.49(0.42-0.56)$ & 0.193 \\
\hline \multicolumn{6}{|l|}{ Allele } \\
\hline PIKR-C & 269 & 151 & 1 (ref.) & 1 (ref.) & \\
\hline PIKR-T & 33 & 51 & $0.36(0.22-0.58)$ & $0.59(0.47-0.73)$ & 0.0001 \\
\hline
\end{tabular}

Abbreviations: $\mathrm{OR}=$ Odds Ratio, $\mathrm{RR}=$ Risk Ratio, $\mathrm{CI}=$ Confidence interval; Statistically significant $p$-values $(p<0.05)$ were indicated with bold numbers.

Table 11. Associations of covariates with PI3KR1 SNP rs706713 genotypes.

\begin{tabular}{|c|c|c|c|c|c|c|c|}
\hline Subjects & $n=101$ & $\mathrm{C} / \mathrm{C}$ & $\mathrm{T} / \mathrm{C}$ & $\mathrm{T} / \mathrm{T}$ & $x^{2}$ & df & $p$-Value \\
\hline \multicolumn{8}{|l|}{ Association with gender } \\
\hline Males & 69 & 42 & 27 & 0 & 4.13 & 2 & 0.126 \\
\hline Females & 32 & 26 & 06 & 0 & & & \\
\hline \multicolumn{8}{|l|}{ Association with Age } \\
\hline Age $>20$ & 17 & 08 & 09 & 0 & 8.47 & 2 & 0.014 \\
\hline Age $>40$ & 84 & 60 & 14 & 0 & & & \\
\hline \multicolumn{8}{|c|}{ Association with RBS mg/dL } \\
\hline RBS $<140$ & 27 & 14 & 13 & 0 & 2.13 & 2 & 0.344 \\
\hline RBS > 140 & 51 & 35 & 16 & 0 & & & \\
\hline \multicolumn{8}{|c|}{ Association with Cholesterol mg/dL } \\
\hline Cholesterol < 200 & 53 & 32 & 21 & 0 & 0.02 & 2 & 0.990 \\
\hline Cholesterol > 200 & 29 & 18 & 11 & 0 & & & \\
\hline \multicolumn{8}{|c|}{ Association with HDL-C mg/dL } \\
\hline $\mathrm{HDL}<55$ & 69 & 44 & 25 & 0 & 0.84 & 2 & 0.657 \\
\hline HDL > 55 & 13 & 10 & 03 & 0 & & & \\
\hline \multicolumn{8}{|c|}{ Association with LDL-C mg/dL } \\
\hline $\mathrm{LDL}<100$ & 31 & 21 & 10 & 0 & 0.0 & 2 & 1 \\
\hline LDL $>100$ & 52 & 35 & 17 & 0 & & & \\
\hline \multicolumn{8}{|c|}{ Association with TG mg/dL } \\
\hline $\mathrm{TG}<200 \mathrm{mg}$ & 63 & 43 & 20 & 0 & 0.17 & 2 & 0.918 \\
\hline TG $>200 \mathrm{mg}$ & 19 & 12 & 07 & 0 & & & \\
\hline \multicolumn{8}{|l|}{ Association with HBA1c \% } \\
\hline HBA1c $<6$ mg & 02 & 1 & 1 & 0 & 0.28 & 2 & 0.869 \\
\hline HBA1c $>6 \mathrm{mg}$ & 99 & 67 & 32 & 0 & & & \\
\hline \multicolumn{8}{|c|}{ Association with Vitamin D ng/mL } \\
\hline Vit.D $<30$ & 15 & 11 & 04 & 0 & 0.6 & 2 & 0.740 \\
\hline Vit.D > 30 & 15 & 09 & 06 & 0 & & & \\
\hline
\end{tabular}


Results indicated that there was a significant differences in the rs3730089 SNP genotype (GG, GA, AA) between cases $(09,49,42)$ and controls $(18,69,35)$ with a $p$-value $=0.03$ (Table 12). The A allele was associated with T2D with OR $(95 \% \mathrm{CI})=1.49(1.01-2.21)$, $\mathrm{RR}=1.19(1.01-1.41), p$-value $=0.04$ (Table 13). We could not see significant differences in genotype distribution of cases with different parameters (Table 14).

Table 12. Genotype frequency of PI3KR1 SNP rs3730089 G > A polymorphism of study cohorts.

\begin{tabular}{lcccccc}
\hline Variables/Genotype & G/G & G/A & A/A & $\chi^{2}$ & df & $p$-Value \\
\hline PIKR patients & $9(9 \%)$ & $49(49 \%)$ & $42(42 \%)$ & 6.71 & 2 & $\mathbf{0 . 0 3}$ \\
\hline Controls & $18(14.8 \%)$ & $69(56.5 \%)$ & $35(28.7 \%)$ & & & \\
\hline
\end{tabular}

Statistically significant $p$-values $(p<0.05)$ were indicated with bold numbers.

Table 13. Association of PI3KR1 SNP rs3730089 G > A gene variation with PIKR.

\begin{tabular}{|c|c|c|c|c|c|}
\hline $\begin{array}{c}\text { Mode of } \\
\text { Inheritance }\end{array}$ & $\begin{array}{c}\text { Cases } \\
(n=100)\end{array}$ & Controls $(n=101)$ & OR (95\% CI) & RR (95\% CI) & $p$-Value \\
\hline \multicolumn{6}{|l|}{ Co-dominant } \\
\hline PIKR-GG & 9 & 18 & 1 (ref.) & 1 (ref.) & \\
\hline PIKR-GA & 49 & 69 & $1.42(0.58-3.42)$ & $1.14(0.83-1.54)$ & 0.434 \\
\hline PIKR-AA & 42 & 35 & $2.4(0.95-6.0)$ & $1.46(1.02-2.1)$ & 0.061 \\
\hline \multicolumn{6}{|l|}{ Dominant } \\
\hline PIKR-GG & 9 & 18 & 1 (ref.) & 1 (ref.) & \\
\hline PIKR-(GA + AA) & 91 & 104 & $1.75(0.74-4.08)$ & $1.25(0.92-1.68)$ & 0.196 \\
\hline \multicolumn{6}{|l|}{ Recessive } \\
\hline PIKR-(GG + GA) & 58 & 87 & 1 (ref.) & 1 (ref.) & \\
\hline PIKR-AA & 42 & 35 & $1.8(1.03-3.14)$ & $1.32(0.99-1.74)$ & 0.039 \\
\hline \multicolumn{6}{|l|}{ Allele } \\
\hline PIKR-G & 67 & 105 & 1 (ref.) & 1 (ref.) & \\
\hline PIKR-A & 133 & 139 & 1.49 (1.01-2.21) & $1.19(1.01-1.41)$ & 0.040 \\
\hline
\end{tabular}

Abbreviations: $\mathrm{OR}=$ Odds Ratio, $\mathrm{RR}=$ Risk Ratio, $\mathrm{CI}=$ Confidence interval; Statistically significant $p$-values $(p<0.05)$ were indicated with bold numbers.

Table 14. Associations of covariates with PI3KR1 SNP rs3730089 genotypes.

\begin{tabular}{|c|c|c|c|c|c|c|c|}
\hline Subjects & $n=100$ & G/G & G/A & $\mathbf{A} / \mathbf{A}$ & $x^{2}$ & df & $p$-Value \\
\hline \multicolumn{8}{|l|}{ Association with gender } \\
\hline Males & 62 & 3 & 30 & 29 & 4.04 & 2 & 0.132 \\
\hline Females & 38 & 6 & 19 & 13 & & & \\
\hline \multicolumn{8}{|l|}{ Association with Age } \\
\hline Age $>20$ & 15 & 0 & 6 & 9 & 3.24 & 2 & 0.197 \\
\hline Age $>40$ & 85 & 9 & 43 & 33 & & & \\
\hline \multicolumn{8}{|c|}{ Association with RBS mg/dL } \\
\hline RBS $<140 \mathrm{mg}$ & 25 & 3 & 8 & 14 & 2.25 & 2 & 0.324 \\
\hline RBS > $140 \mathrm{mg}$ & 54 & 5 & 27 & 22 & & & \\
\hline
\end{tabular}


Table 14. Cont.

\begin{tabular}{|c|c|c|c|c|c|c|c|}
\hline Subjects & $n=100$ & G/G & G/A & $\mathbf{A} / \mathbf{A}$ & $x^{2}$ & df & $p$-Value \\
\hline \multicolumn{8}{|c|}{ Association with Cholesterol mg/dL } \\
\hline Cholesterol < 200 & 50 & 5 & 21 & 24 & 0.82 & 2 & 0.663 \\
\hline Cholesterol > 200 & 31 & 2 & 16 & 13 & & & \\
\hline \multicolumn{8}{|c|}{ Association with HDL-C mg/dL } \\
\hline $\mathrm{HDL}<55$ & 70 & 7 & 32 & 31 & 1.32 & 2 & 0.516 \\
\hline HDL > 55 & 11 & 0 & 5 & 6 & & & \\
\hline \multicolumn{8}{|c|}{ Association with LDL-C mg/dL } \\
\hline LDL $<100$ & 27 & 4 & 11 & 12 & 2.02 & 2 & 0.364 \\
\hline LDL $>100$ & 54 & 3 & 26 & 25 & & & \\
\hline \multicolumn{8}{|c|}{ Association with TG mg/dL } \\
\hline $\mathrm{TG}<200 \mathrm{mg}$ & 61 & 6 & 24 & 31 & 4.01 & 2 & 0.134 \\
\hline TG $>200 \mathrm{mg}$ & 20 & 1 & 13 & 6 & & & \\
\hline \multicolumn{8}{|c|}{ Association with HBA1c \% } \\
\hline HBA1c $<6$ mg & 1 & 0 & 1 & 0 & 1.07 & 2 & 0.585 \\
\hline HBA1c $>6 \mathrm{mg}$ & 98 & 9 & 47 & 42 & & & \\
\hline \multicolumn{8}{|c|}{ Association with Vitamin D ng/mL } \\
\hline Vit.D $<30$ & 14 & 2 & 7 & 5 & 0.94 & 2 & 0.625 \\
\hline Vit.D > 30 & 17 & 2 & 6 & 9 & & & \\
\hline
\end{tabular}

\section{Discussion}

$\mathrm{DM}$ is one of the important causes of morbidity and mortality all over the world. It has serious impact on public health system as well as socioeconomic status of patients and their families. The incidence and prevalence rates of DM in KSA were increased [23]. MiRNAs are short, noncoding, single stranded RNA molecules that have been involved in crucial biological process such as development, metabolism, differentiation and apoptosis [15]. Gene variations of MiRNAs were associated with many diseases including T2D [11,24]. The MiR-126 gene is found within the gene of the epidermal growth factor-like protein 7 (EGFL7) in chromosome 9q34.3 [25]. This polymorphism has been reported to be associated with ischemic stroke in Chinese population [25]. In addition, reduced mir126 plasma levels have been suggested as a biomarker for early identification of individual susceptibility to T2D [26]. Our results indicated that Mir126 rs4636297 genotypes distribution was significantly different ( $p$-value $<0.05$ ) between T2D cases and the control (Table 3$)$. The GG genotype of the rs4636297 was associated with T2D (Table 4). The G of the rs4636297 allele was also associated with T2D (Table 4). This result maybe in partial agreement with previous studies suggested that the miR-126 rs4636297 SNP was associated with diabetic retinopathy in different populations $[27,28]$. In addition, results showed that there were significant differences $(p$-value $<0.05)$ in the lipid profile in cases with normal and cases with abnormal lipid profile (Table 5). This result may also be consistent with studies suggested that miR-126 is involved lipid metabolism $[29,30]$ because there was an association between miR-126 and LDL-C circulating levels [30]. This rs4636297 SNP may reduce the amount of miR-126 in plasma; the reduced miR-126 was described as an early indication of individual risk to T2D [26]. However, the effect of this SNP on miR-126 remains to be investigated in future studies.

PI3-kinases are family of lipid kinases that catalyze the addition of phosphate to the 3-OH position of cellular membrane inositol lipids [31]. PI3-kinases are important for several physiological processes such as metabolism, proliferation, survival and growth [31,32]. The PI3-kinases are heterodimer enzymes composed of regulatory subunit (e.g., p85 $\alpha$, 
p85 $\beta, p 55 \alpha, p 50 \alpha$, or p55 $\gamma$ ) and catalytic subunits (e.g., p110 $\alpha, \beta$ or $\delta$ ) [33]. There are several isoforms of the PI3-kinases regulatory and catalytic subunits [31,33]. This enzyme is very important for insulin metabolic action [31]. It has been reported that construction of dysfunctional PI3-kinases or pharmacological inhibition of PI3-kinases resulted in dysregulation of glucose transport, synthesis of lipid and glycogenesis [31]. Furthermore, abolishment of PI3-kinase stimulation by insulin in animal model resulted in increased body fat and T2D development [31], whereas deletion of regulatory subunit of the PI3kinases in muscles resulted in impairment of insulin signaling, deceased uptake of glucose and increased glucose plasma levels $[19,34]$. Our results indicated that there was a significant difference ( $p$-value $<0.05$ ) in the SNP rs7713645 (in PIKR intron) genotype distribution between cases and controls (Table 6), and that the CA and CC genotypes and the $C$ allele of the rs7713645 were associated with T2D (Table 7). Results also showed that there is a significant difference ( $p$-value $<0.05)$ in SNP rs7713645 and the genotype distributions between cases with normal and cases with abnormal lipid profile (Table 8), and there was a significant differences in rs7713645 genotype distribution between cases with age $>20$ and cases with age $>40$ years (Table 8). This result is consistent with previous studies reported that the SNP rs7713645 is associated with higher MBI, increased body fat, and higher fasting glucose levels [35]. This result is also in agreement with a study that reported an association of rs7713645 with T2D in Turkish population [36].

Results indicated that there was a significant difference $(p$-value $<0.05)$ in the rs706713 (in PIKR exon1) genotype distribution between cases and controls (Table 9). The rs706713 CT genotype and the T allele were associated with T2D (Table 10). There was also a significant difference between cases with age $>20$ and cases with age $>40$ years (Table 11). The SNP rs706713 is a silent mutation, i.e., there is no change in the amino acid (Tyr73Tyr) [36]. We do not have explanation why there was a significant difference in rs706713 genotype distribution between cases and controls, and a significant difference between cases with age $>20$ and cases with age $>40$ years old. However, this result is consistent with Karadoğan et al. who indicated association of rs706713 with T2D in Turkish population [36].

Results also showed that the exon SNP rs3730089 (Met326Ile, in exon 6) genotype distribution were significantly different between cases and control (Table 12) and that the A allele of the rs3730089 was associated with T2D (Table 13). A previous study [37] examined the expression of PI3-kinase regulatory subunit $(\mathrm{p} 85 \alpha)$ and protein-protein interaction of the insulin receptor substrate (IRS-1) with p85 $\alpha$ using the yeast two hybrid assay $[37,38]$. It was reported that the SNP rs3730089 (Met326Ile) resulted in reduced p85 $\alpha$ expression but increased binding between p85 $\alpha$ and IRS1 [37], and therefore the rs3730089 may have minor impact on insulin signaling and glucose uptake [37]. This result is consistent with previous studies showed potential association of rs3730089 SNP with T2D [36] and insulin signaling [37]. However, another study reported no association of rs3730089 SNP with T2D in north Indian population [39]. This difference may be due to different sample sizes or different populations. We did no find significant differences in rs3730089 genotype distribution of cases with different parameters (Table 14). This is probably due to the small sample size used in this study.

Limitations of this cross-sectional study include the small sample size and the blood samples were collected from patients one time and maybe after the blood biochemistry was already controlled.

Future longitudinal studies with larger sample sizes and in different populations to examine the effects of both the miR-126 SNP and one or more PIKR risk alleles on susceptibility to T2D, T2D complications and lipid metabolism are strongly recommended. In addition, further-protein protein interactions studies using the techniques such as yeasttwo hybrid, phage display, x-ray crystallography, protein NMR spectroscopy $[38,40-43]$ are required to examine the effect of SNPs in PI3KR1 gene on the PI3K structure, function and interaction with other proteins.

In summary, our study indicated that the Mir126 rs4636297 was associated with T2D. Results also showed that there was a significant difference in rs4636297 in genotype 
distribution between cases with normal and cases with abnormal lipid profile. Results also showed that the PI3KR1 SNPs rs7713645, rs3730089 were associated with T2D. The PI3KR1 SNP rs7713645 may also be associated with abnormal lipid profile. These results require further verification in future studies with larger sample sizes and in different populations.

Author Contributions: Conceptualization, all authors, R.M., I.E., F.M.A.D., M.A.A., A.I.A., J.B., A.T.B., M.M.M., H.M., A.H., P.K.D.; methodology, R.M. and I.E.; validation, R.M. and I.E.; formal analysis, R.M. and I.E.; investigation, All authors.; resources, All authors.; data curation, R.M. and I.E.; writing—original draft preparation, R.M. and I.E.; writing—review and editing, I.E.; visualization, All authors; supervision, R.M., I.E., F.M.A.D.; project administration, I.E.; funding acquisition, I.E. All authors have read and agreed to the published version of the manuscript.

Funding: This project is funded by the University of Tabuk, Deanship of scientific research (DSR), project No 0012-1441-S for IE and colleagues.

Institutional Review Board Statement: This study was approved by the research and studies department, directorate of health affairs, Taif, approval No. 229, and by the research ethics committee of the armed forces hospitals, northwestern region, Tabuk, approval No. R \& REC2016-115.

Informed Consent Statement: Informed consent was obtained from all subjects involved in the study. Data Availability Statement: Not applicable.

Acknowledgments: We thank T2D patients and healthy controls for their participation in this study.

Conflicts of Interest: The authors declare no conflict of interest.

\section{References}

1. American Diabetes Association. Diagnosis and classification of diabetes mellitus. Diabetes Care 2013, 36, S67-S74. [CrossRef] [PubMed]

2. Lin, X.; Xu, Y.; Pan, X.; Xu, J.; Ding, Y.; Sun, X.; Song, X.; Ren, Y.; Shan, P.-F. Global, regional, and national burden and trend of diabetes in 195 countries and territories: An analysis from 1990 to 2025. Sci. Rep. 2020, 10, 14790. [CrossRef] [PubMed]

3. Al Dawish, M.A.; Robert, A.A.; Braham, R.; Al Hayek, A.A.; Al Saeed, A.; Ahmed, R.A.; Al Sabaan, F.S. Diabetes Mellitus in Saudi Arabia: A Review of the Recent Literature. Curr. Diabetes Rev. 2016, 12, 359-368. [CrossRef] [PubMed]

4. Beckman, J.A.; Creager, M.A. Vascular Complications of Diabetes. Circ. Res. 2016, 118, 1771-1785. [CrossRef]

5. Burrack, A.L.; Martinov, T.; Fife, B.T. T Cell-Mediated Beta Cell Destruction: Autoimmunity and Alloimmunity in the Context of Type 1 Diabetes. Front. Endocrinol. 2017, 8, 343. [CrossRef]

6. Czech, M.P. Insulin action and resistance in obesity and type 2 diabetes. Nat. Med. 2017, 23, 804-814. [CrossRef]

7. Dendup, T.; Feng, X.; Clingan, S.; Astell-Burt, T. Environmental Risk Factors for Developing Type 2 Diabetes Mellitus: A Systematic Review. Int. J. Environ. Res. Public Health 2018, 15, 78. [CrossRef]

8. Elfaki, I.; Mir, R.; Abu-Duhier, F.M.; Jha, C.K.; Ahmad Al-Alawy, A.I.; Babakr, A.T.; Habib, S.A.E.H. Analysis of the Potential Association of Drug-Metabolizing Enzymes CYP2C9*3 and CYP2C19*3 Gene Varia-tions With Type 2 Diabetes: A Case-Control Study. Curr. Drug Metab. 2020, 21, 1152-1160. [CrossRef]

9. Elfaki, I.; Almutairi, F.M.; Mir, R.; Khan, R.; Abu-duhier, F. Cytochrome P450 CYP1B1*2 gene and its association with T2D in Tabuk population, Northwestern region of Saudi Arabia. Asian J. Pharm. Clin. Res. 2018, 11, 55-59. [CrossRef]

10. Xue, A.; Wu, Y.; Zhu, Z.; Zhang, F.; Kemper, K.E.; Zheng, Z.; Yengo, L.; Lloyd-Jones, L.R.; Sidorenko, J.; Wu, Y.; et al. Genome-wide association analyses identify 143 risk variants and putative regulatory mechanisms for type 2 diabetes. Nat. Commun. 2018, 9 , 2941. [CrossRef]

11. Elfaki, I.; Mir, R.; Mir, M.M.; AbuDuhier, F.M.; Babakr, A.T.; Barnawi, J. Potential Impact of MicroRNA Gene Polymorphisms in the Pathogenesis of Diabetes and Atherosclerotic Cardiovascular Disease. J. Pers. Med. 2019, 9, 51. [CrossRef]

12. Jha, C.K.; Mir, R.; Elfaki, I.; Javid, J.; Babakr, A.; Banu, S.; Chahal, S.M.S. Evaluation of the Association of Omentin 1 rs2274907 $\mathrm{A}>\mathrm{T}$ and rs2274908 G>A Gene Polymorphisms with Coronary Artery Disease in Indian Population: A Case Control Study. J. Pers. Med. 2019, 9, 30. [CrossRef]

13. Elfaki, I.; Mir, R.; Almutairi, F.M.; Abu Duhier, F.M. Cytochrome P450: Polymorphisms and Roles in Cancer, Diabetes and Atherosclerosis. Asian Pac. J. Cancer Prev. 2018, 19, 2057-2070. [CrossRef]

14. Jha, C.K.; Mir, R.; Elfaki, I.; Banu, S.; Chahal, S.M.S. LDLR Gene Polymorphisms (rs5925 and rs1529729) Are Associated with Susceptibility to Coronary Artery Disease in a South Indian Population. Med. Sci. 2019, 7, 80.

15. Pordzik, J.; Jakubik, D.; Jarosz-Popek, J.; Wicik, Z.; Eyileten, C.; De Rosa, S.; Indolfi, C.; Siller-Matula, J.M.; Czajka, P.; Postula, M. Significance of circulating microRNAs in diabetes mellitus type 2 and platelet reactivity: Bioinformatic analysis and review. Cardiovasc. Diabetol. 2019, 18, 113. [CrossRef] 
16. Zampetaki, A.; Kiechl, S.; Drozdov, I.; Willeit, P.; Mayr, U.; Prokopi, M.; Mayr, A.; Weger, S.; Oberhollenzer, F.; Bonora, E.; et al. Plasma MicroRNA Profiling Reveals Loss of Endothelial MiR-126 and Other MicroRNAs in Type 2 Diabetes. Circ. Res. 2010, 107, 810-817. [CrossRef]

17. Liu, Y.; Gao, G.; Yang, C.; Zhou, K.; Shen, B.; Liang, H.; Jiang, X. The Role of Circulating MicroRNA-126 (miR-126): A Novel Biomarker for Screening Prediabetes and Newly Diagnosed Type 2 Diabetes Mellitus. Int. J. Mol. Sci. 2014, 15, 10567-10577. [CrossRef]

18. Massignam, E.T.; Dieter, C.; Pellenz, F.M.; Assmann, T.S.; Crispim, D. Involvement of miR-126 rs4636297 and miR-146a rs2910164 polymorphisms in the susceptibility for diabetic retinopathy: A case-control study in a type 1 diabetes population. Acta Ophthalmol. 2021, 99, 461. [CrossRef]

19. Di Zazzo, E.; Feola, A.; Zuchegna, C.; Romano, A.; Donini, C.F.; Bartollino, S.; Frunzio, R.; Laccetti, P.; Di Domenico, M.; Porcellini, A. The p85 regulatory subunit of PI3K mediates cAMP-PKA and insulin biological effects on MCF-7 cell growth and motility. Sci. World J. 2014, 2014, 565839. [CrossRef]

20. Burke, J.E.; Vadas, O.; Berndt, A.; Finegan, T.; Perisic, O.; Williams, R.L. Dynamics of the phosphoinositide 3-kinase p110delta interaction with p85alpha and membranes reveals aspects of regulation distinct from p110alpha. Structure 2011, 19, 1127-1137. [CrossRef]

21. Hopkins, B.D.; Goncalves, M.D.; Cantley, L.C. Insulin-PI3K signalling: An evolutionarily insulated metabolic driver of cancer. Nat. Rev. Endocrinol. 2020, 16, 276-283. [CrossRef]

22. Huang, X.; Liu, G.; Guo, J.; Su, Z. The PI3K/AKT pathway in obesity and type 2 diabetes. Int. J. Biol. Sci. 2018, 14, 1483-1496. [CrossRef]

23. Alotaibi, A.; Perry, L.; Gholizadeh, L.; Al-Ganmi, A. Incidence and prevalence rates of diabetes mellitus in Saudi Arabia: An overview. J. Epidemiol. Glob. Health 2017, 7, 211-218. [CrossRef]

24. Ding, H.-X.; Lv, Z.; Yuan, Y.; Xu, Q. MiRNA Polymorphisms and Cancer Prognosis: A Systematic Review and Meta-Analysis. Front. Oncol. 2018, 8, 596. [CrossRef]

25. Xiang, Y.; Guo, J.; Peng, Y.-F.; Tan, T.; Huang, H.-T.; Luo, H.-C.; Wei, Y.-S. Association of miR-21, miR-126 and miR-605 gene polymorphisms with ischemic stroke risk. Oncotarget 2017, 8, 95755-95763. [CrossRef]

26. Zhang, T.; Lv, C.; Li, L.; Chen, S.; Liu, S.; Wang, C.; Su, B. Plasma miR-126 is a potential biomarker for early prediction of type 2 diabetes mellitus in susceptible individuals. Biomed. Res. Int. 2013, 2013, 761617. [CrossRef]

27. McAuley, A.K.; Dirani, M.; Connell, P.P.; Lamoureux, E.L.; Wang, J.J.; Hewitt, A.W. A genetic variant regulating miR-126 is associated with sight threatening diabetic retinopathy. Diabetes Vasc. Dis. Res. 2015, 12, 133-138. [CrossRef]

28. Rezai, M.; Shahbazi, S.; Mansournia, N. Plasma miR-126 levels and its genomic polymorphism SNP rs4636297 in Type 2 dia-betes. Asia Pac. J. Mol. Biol. Biotechnol. 2020, 28, 27-33. [CrossRef]

29. Chu, M.; Zhao, Y.; Feng, Y.; Zhang, H.; Liu, J.; Cheng, M.; Li, L.; Shen, W.; Cao, H.; Li, Q.; et al. MicroRNA-126 participates in lipid metabolism in mammary epithelial cells. Mol. Cell. Endocrinol. 2017, 454, 77-86. [CrossRef]

30. Sun, X.; Zhang, M.; Sanagawa, A.; Mori, C.; Ito, S.; Iwaki, S.; Satoh, H.; Fujii, S. Circulating microRNA-126 in patients with coronary artery disease: Correlation with LDL cholesterol. Thromb. J. 2012, 10, 16. [CrossRef]

31. Winnay, J.N.; Boucher, J.; Mori, M.; Ueki, K.; Kahn, C.R. A regulatory subunit of phosphoinositide 3-kinase increases the nuclear accumulation of X-box-binding protein-1 to modulate the unfolded protein response. Nat. Med. 2010, 16, 438-445. [CrossRef] [PubMed]

32. Akinleye, A.; Avvaru, P.; Furqan, M.; Song, Y.; Liu, D. Phosphatidylinositol 3-kinase (PI3K) inhibitors as cancer therapeutics. J. Hematol. Oncol. 2013, 6, 88. [CrossRef] [PubMed]

33. Backer, J.M. The Regulation of Class IA PI 3-Kinases by Inter-Subunit Interactions. Curr. Top. Microbiol. Immunol. 2010, 346, 87-114. [CrossRef] [PubMed]

34. Maffei, A.; Lembo, G.; Carnevale, D. PI3Kinases in Diabetes Mellitus and Its Related Complications. Int. J. Mol. Sci. 2018, 19, 4098. [CrossRef]

35. Jamshidi, Y.; Snieder, H.; Wang, X.; Pavitt, M.J.; Spector, T.D.; Carter, N.D.; O’Dell, S.D. Phosphatidylinositol 3-kinase p85 $\alpha$ regulatory subunit gene PIK3R1 haplotype is associated with body fat and serum leptin in a female twin population. Diabetologia 2006, 49, 2659-2667. [CrossRef]

36. Karadoğan, A.H.; Arikoglu, H.; Gokturk, F.; Iscioglu, F.; Ipekçi, S.H. PIK3R1 gene polymorphisms are associated with type 2 diabetes and related features in the Turkish population. Adv. Clin. Exp. Med. 2018, 27, 921-927. [CrossRef]

37. Almind, K.; Delahaye, L.; Hansen, T.; Van Obberghen, E.; Pedersen, O.; Kahn, C.R. Characterization of the Met326Ile variant of phosphatidylinositol 3-kinase p85. Proc. Natl. Acad. Sci. USA 2002, 99, 2124-2128. [CrossRef]

38. Elfaki, I.; Bayer, P.; Mueller, J.W. A potential transcriptional regulator is out-of-frame translated from the metallothionein 2A messenger RNA. Anal. Biochem. 2011, 409, 159-161. [CrossRef]

39. Rai, E.; Sharma, S.; Kaul, S.; Jain, K.; Matharoo, K.; Bhanwer, A.S.; Bamezai, R.N.K. The Interactive Effect of SIRT1 Promoter Region Polymorphism on Type 2 Diabetes Susceptibility in the North Indian Population. PLoS ONE 2012, 7, e48621. [CrossRef]

40. Elfaki, I.; Knitsch, A.; Matena, A.; Bayer, P. Identification and characterization of peptides that bind the PPIase domain of Parvulin17. J. Pept. Sci. 2013, 19, 362-369. [CrossRef] 
41. Mompeán, M.; Romano, V.; Pantoja-Uceda, D.; Stuani, C.; Baralle, F.E.; Buratti, E.; Laurents, D.V. Point mutations in the N-terminal domain of transactive response DNA-binding protein $43 \mathrm{kDa}$ (TDP-43) compromise its stability, dimerization, and functions. J. Biol. Chem. 2017, 292, 11992-12006. [CrossRef]

42. Jaremko, L.; Jaremko, M.; Elfaki, I.; Mueller, J.W.; Ejchart, A.; Bayer, P.; Zhukov, I. Structure and dynamics of the first archaeal parvulin reveal a new functionally important loop in par-vulin-type prolyl isomerases. J. Biol. Chem. 2011, 286, 6554-6565. [CrossRef]

43. Kumar, R.; Moche, M.; Winblad, B.; Pavlov, P.F. Combined X-ray crystallography and computational modeling approach to investigate the Hsp90 C-terminal peptide binding to FKBP51. Sci. Rep. 2017, 7, 14288. [CrossRef] 